\title{
Shear viscosity in microscopic calculations of $A+A$ collisions at energies available at the Nuclotron-based Ion Collider fAcility (NICA)
}

\author{
M. Teslyk $\odot,{ }^{*}$ L. Bravina, O. Panova $\odot,{ }^{*}$ O. Vitiuk $\odot,{ }^{*}$ and E. Zabrodin $\odot^{\dagger}$ \\ Department of Physics, University of Oslo, PB 1048 Blindern, N-0316 Oslo, Norway
}

(Received 8 October 2019; published 8 January 2020)

\begin{abstract}
Time evolution of shear viscosity $\eta$, entropy density $s$, and their ratio $\eta / s$ in the central area of central goldgold collisions at energies available at the Nuclotron-based Ion Collider fAcility (NICA) are studied within the ultrarelativistic quantum molecular dynamics (UrQMD) transport model. The extracted values of energy density, net baryon density, and net strangeness density are used as input to (i) the statistical model of an ideal hadron gas to define temperature, baryochemical potential, and strangeness chemical potential, and to (ii) a UrQMD box with periodic boundary conditions to study the relaxation process of highly excited matter. During the relaxation stage, the shear viscosity is determined in the framework of the Green-Kubo approach. The procedure is performed for each of 20 time slices, corresponding to conditions in the central area of the fireball at times from 1 to $20 \mathrm{fm} / c$. For all tested energies the ratio $\eta / s$ reaches its minimum, $(\eta / s)_{\min } \approx 0.3$ at $t \approx 5 \mathrm{fm} / c$. Then it increases up to the late stages of the system evolution. This rise is accompanied by the drop of both temperature and strangeness chemical potential and by the increase of baryochemical potential.
\end{abstract}

DOI: 10.1103/PhysRevC.101.014904

\section{INTRODUCTION}

Relativistic heavy-ion collisions have been intensively studied both theoretically and experimentally to obtain information about the properties of highly excited nuclear matter. To date, these collisions are the only means to study the conditions of early Universe in the laboratory, thus leading to the term "little big bang" [1]. According to the theoretical estimates and lattice quantum chromodynamics (IQCD) calculations, nuclear matter under certain extreme conditions should experience a deconfinement phase transition into a new phase of matter, a quark-gluon plasma (QGP). The expanding hot fireball should, however, rapidly cool off, and the plasma will undergo hadronization. Experiments show that in heavyion collisions at the ultrarelativistic energies of the Relativistic Heavy Ion Collider (RHIC), $\sqrt{s}=200 \mathrm{GeV}$, and of the Large Hadron Collider (LHC), $\sqrt{s}=2.76$ and $5.02 \mathrm{TeV}$, there is a crossover type of the phase transition. In contrast, at much lower energies the transition might be of the first order. In this case the line of the first-order phase transition in the nuclear phase diagram ends up in the tricritical point, where the transition becomes of second order. The search for the tricritical point is in the agenda of experiments with heavy-ion beams at the forthcoming Nuclotron-based Ion Collider fAcility (NICA) and the Facility for Antiproton and Ion Research (FAIR), and within the beam energy scan (BES) program at RHIC. Therefore, one has to look for the observable most

\footnotetext{
*Also at Taras Shevchenko National University of Kyiv, UA-01033 Kyiv, Ukraine.

${ }^{\dagger}$ Also at Skobeltsyn Institute of Nuclear Physics,Moscow State University, RU-119991 Moscow, Russia.
}

sensitive to the QGP-hadrons transition. One such observable is the ratio of shear viscosity $\eta$ to entropy density $s, \eta / s$. This ratio drops to a minimum at critical temperatures for all known substances [2], and in relativistic heavy-ion collisions it is expected to be of order of its theoretical lower bound, $1 / 4 \pi$ [3]; for details see, e.g., [4] and references therein.

Despite the interest in this topic, it is still difficult to estimate the value of the ratio exactly due to the high calculation complexity required by QCD simulations. Therefore, various works in the field have explored different approaches and approximations for the conditions expected to prevail near the phase transition; see e.g., [5-14]. For example, in [5] thermodynamic quantities of hadronic matter are studied for a system of light mesons embedded in a box with periodic boundary conditions generated by the ultrarelativistic quantum molecular dynamics (UrQMD) model. A relativistic hadron gas in thermal and chemical equilibrium and with zero baryon and strangeness chemical potentials was considered in [6]. In [11] the authors obtain viscosity $\eta$ by solving the ultrarelativistic Boltzmann transport equation and compare it to the one obtained via the Chapman-Enskog approximation. Recently, the shear viscosity and its ratio to entropy density were calculated for a gas of Hagedorn states [15] with masses up to $10 \mathrm{GeV} / c^{2}$. It was found that, because of the rapid growth of $s$ in the vicinity of Hagedorn limiting temperature $T_{\mathrm{H}}=165 \mathrm{MeV}$, the ratio $\eta / s$ came close to and was even below the bounding $1 / 4 \pi$ given by anti-de Sitter and conformal field theory (AdS-CFT) [3]. Among the other papers on the topic are [16], where viscosity is extracted within the SMASH transport model, and [17], where the UrQMD model was employed for system of nucleons at intermediate temperatures between 10 and $50 \mathrm{MeV}$. In the latter case the nucleons were allowed to experience only elastic collisions. 
Definitely, heavy-ion collisions at energies of NICA and higher are more complex. As mentioned in [18], the ratio $\eta / s$ cannot be constant during the evolution of the fireball. To provide better fits to the experimental data, this ratio should depend on both temperature and chemical potentials. Consequently, it is essential to explore the time dynamics of the ratio $\eta / s$ from the very beginning of a relativistic heavyion collision.

In the present paper we investigate fluctuation relaxation time $\tau$ and shear viscosity $\eta$, as well as its ratio to entropy density, $\eta / s$, for central $\mathrm{Au}+\mathrm{Au}$ collisions calculated in the UrQMD model $[19,20]$ within the NICA energy range. Compared to the previous researches, we study the evolution of $\eta$, $s$, and $\eta / s$ in heavy-ion collisions, where all characteristics are quickly changing, and not, e.g., the temperature dependence of the $\eta / s$ ratio at constant chemical potentials. Investigation of dynamics of the relaxation process in a box with periodic boundary conditions allows us to estimate both the lower and upper bounds of the time interval at different energies, where it is possible to extract $\tau$.

The paper is organized as follows. Section II describes briefly the features of the UrQMD model and the UrQMD box calculations. To extract the thermodynamic quantities, such as temperature $T$, baryochemical potential $\mu_{\mathrm{B}}$ and strangeness chemical potential $\mu_{\mathrm{S}}$, one has to compare microscopic model calculations with the results provided by the statistical model (SM) of an ideal hadron gas with essentially the same degrees of freedom. This model is also explained in Sec. II. The formalism employed to determine the shear viscosity of hot and dense nuclear matter is presented in Sec. III. Section IV contains results of our study, including the time evolution of $\eta$ and $\eta / s$ in the central area of heavy-ion collisions, and dependencies of $\eta / s$ on $T, \mu_{\mathrm{B}}$, and $\mu_{\mathrm{S}}$. Finally, conclusions are drawn in Sec. V.

\section{MODELS EMPLOYED FOR THE ANALYSIS}

In our study of shear viscosity we employ three computational models. The first one is the microscopic transport model UrQMD to calculate $A+A$ collisions at a given energy and get the bulk characteristics of hot and dense nuclear matter, namely, energy density $\varepsilon$, net baryon density $\rho_{\mathrm{B}}$, and net strangeness density $\rho_{\mathrm{S}}$. The second model is the UrQMD box with periodic boundary conditions to study the relaxation process and find the relaxation time $\tau$. Finally, to determine thermodynamic parameters of the equilibrated system, i.e., temperature $T$, baryon chemical potential $\mu_{\mathrm{B}}$, and strangeness chemical potential $\mu_{\mathrm{S}}$, we apply the statistical model of an ideal hadron gas. The main features of all three models are as follows.

\section{A. UrQMD model}

This is a well-known model $[19,20]$ widely used for the analysis of heavy-ion collisions in a broad energy range. UrQMD is based on covariant propagation of hadrons on classical trajectories, stochastic binary interactions of these hadrons if the distance between them is less than $d \leqslant d_{0}=$ $\sqrt{\sigma^{\text {tot }} / \pi}$, where $\sigma^{\text {tot }}$ is the total cross section, formation and decay of resonances, and, when a certain collision energy limit is exceeded, formation and subsequent fragmentation of specific colored objects, strings. For the treatment of strings UrQMD employs classical Lund model [21]. As independent degrees of freedom the model considers 55 different baryon states with masses up to $m_{\mathrm{B}}^{\max } \leqslant 2.25 \mathrm{GeV} / c^{2}$ and 39 different meson states, including the charmed ones. The list of particles is supplemented by corresponding antiparticles and isospin-projected states. Cross sections of hadron-hadron $(h h)$ interactions are taken from the available experimental data [22]. If this information is missing, the model relies on the unitarity, the additive quark model, and detailed balance considerations.

\section{B. Calculation of nuclear infinite matter: UrQMD box}

The box with finite volume and periodic boundary conditions serves to simulate the properties of infinite nuclear matter [23,24]. All particle interactions assumed in UrQMD are allowed in the box as well. However, if any particle leaves the box, another particle with absolutely identical parameters enters it, thus ensuring the preservation of initial energy density, net baryon density, and net strangeness density in the box. The initial state in the box can be generated as mixture of baryons and antibaryons, or a baryon-free gas of mesons, or even a system of strings and resonances. In the case of nonzero net baryon charge and zero net strangeness it is convenient to initialize the box containing neutrons and protons only. All nucleons can be uniformly distributed in the space, whereas their momenta are randomly distributed in a Fermi sphere and then rescaled to ensure the required energy density. Note also that relaxation to equilibrium in the box proceeds much longer compared to, e.g., that in the central cell in heavy-ion collisions [24]. In an open-system-like cell, the most energetic particles leave it earlier, and the whole system is cooling down. In a closed-system-like box, one has to wait until the kinetic energy of the most "hot" particles will be redistributed among other particles and also converted to the mass of newly produced hadrons.

Finally, we have to determine temperature and chemical potentials in the system. This is done by multiple fits of hadron abundances and energy spectra in UrQMD to those calculated within the statistical model.

\section{Statistical model of ideal hadron gas}

If the system of hadrons containing $1 \leqslant i \leqslant n$ different species is in equilibrium at temperature $T$, all many-particle correlations in it are reduced to a set of distribution functions (in system of natural units $c=\hbar=k_{B}=1$ )

$$
f\left(p, m_{i}\right)=\left[\exp \left(\frac{\epsilon_{i}-\mu_{i}}{T}\right)+C\right]^{-1} .
$$

Here $C=+1$ for fermions and $C=-1$ for baryons, and $p, m_{i}, \epsilon_{i}$, and $\mu_{i}$ are hadron momentum, mass, energy, and chemical potential, respectively. The last depends on chemical potentials assigned to baryon charge $B_{i}$, strangeness content 
$S_{i}$, and electric charge $Q_{i}$ of $i$ th hadron. However, the chemical potential $\mu_{\mathrm{Q}}$ of electric charge is usually much smaller compared to baryochemical potential $\mu_{\mathrm{B}}$ and strangeness chemical potential $\mu_{\mathrm{S}}$. Therefore, we will consider the linear combination of two terms for the full chemical potential of a hadron:

$$
\mu_{i}=B_{i} \mu_{\mathrm{B}}+S_{i} \mu_{\mathrm{S}}
$$

The partial number density $n_{i}$, the energy density $\varepsilon_{i}$, and the entropy density $s_{i}$ read

$$
\begin{gathered}
n_{i}=\frac{g_{i}}{2 \pi^{2}} \int_{0}^{\infty} f\left(p, m_{i}\right) p^{2} d p \\
\varepsilon_{i}=\frac{g_{i}}{2 \pi^{2}} \int_{0}^{\infty} \sqrt{p^{2}+m_{i}^{2}} f\left(p, m_{i}\right) p^{2} d p \\
s_{i}=-\frac{g_{i}}{2 \pi^{2}} \int_{0}^{\infty} f\left(p, m_{i}\right)\left[\ln f\left(p, m_{i}\right)-1\right] p^{2} d p
\end{gathered}
$$

where $g_{i}$ is the spin-isospin degeneracy factor. The values of $T, \mu_{\mathrm{B}}$, and $\mu_{\mathrm{S}}$ should satisfy the set of nonlinear equations

$$
\begin{aligned}
\varepsilon & =\sum_{i} \varepsilon_{i}\left(T, \mu_{\mathrm{B}}, \mu_{\mathrm{S}}\right), \\
\rho_{B} & =\sum_{i} B_{i} n_{i}\left(T, \mu_{\mathrm{B}}, \mu_{\mathrm{S}}\right), \\
\rho_{S} & =\sum_{i} S_{i} n_{i}\left(T, \mu_{\mathrm{B}}, \mu_{\mathrm{S}}\right),
\end{aligned}
$$

where $\varepsilon, \rho_{\mathrm{B}}$, and $\rho_{\mathrm{S}}$ are taken as input from microscopic model calculations.

\section{SHEAR VISCOSITY DETERMINATION PROCEDURE}

We calculate central $\mathrm{Au}+\mathrm{Au}$ collisions in the laboratory frame at energies $E_{\mathrm{lab}}=10 \mathrm{~A}, 20 \mathrm{~A}, 30 \mathrm{~A}$, and $40 \mathrm{~A} \mathrm{GeV}$, corresponding to $\sqrt{s}$ from 4.5 to $8.8 \mathrm{GeV}$ in the center-of-mass frame. From the whole system the central cell with volume $5 \times 5 \times 5=125 \mathrm{fm}^{3}$ is selected. Then, the energy density $\varepsilon$, the net baryon density $\rho_{\mathrm{B}}$, and the net strangeness density $\rho_{\mathrm{S}}$ in the cell are extracted at times $t_{\text {cell }}=1-20 \mathrm{fm} / c$ with the time step of $1 \mathrm{fm} / c$. In order to minimize statistical errors an ensemble of $51200 \mathrm{Au}+\mathrm{Au}$ central collisions at each energy has been generated.

The extracted data are inserted in the statistical model of the ideal hadron gas to obtain temperature $T$, entropy density $s_{\mathrm{sm}}$, baryon chemical potential $\mu_{\mathrm{B}}$, and strangeness chemical potential $\mu_{\mathrm{s}}$. After that we start UrQMD box calculations. The box with volume $V=10 \times 10 \times 10=1000 \mathrm{fm}^{3}$ is initialized with the same values of $\varepsilon, \rho_{\mathrm{B}}$, and $\rho_{\mathrm{S}}$ as extracted from the cell analysis. Baryon density is provided by protons and neutrons taken in equal proportion, $N_{p}: N_{n}=1: 1$. Nonzero strangeness density is generated by the admixture of kaons. The box data are analyzed for times $t_{\text {box }}=1-1000 \mathrm{fm} / c$ with the time step $1 \mathrm{fm} / c$. The box ensemble consists of 12800 box simulations for each of 80 points.

To extract $\eta$ the Green-Kubo [25,26] formalism was used. The formalism requires the existence of an equilibrated state in the medium in order to provide exponential damping of deviations from the equilibrium with time. Thus, the verification of equilibrium or of exponential damping of fluctuations is the necessary condition to be checked.

From the Green-Kubo formalism it follows that shear viscosity $\eta$ may be defined as

$$
\eta\left(t_{0}\right)=\frac{V}{T} \int_{t_{0}}^{\infty} d t\left\langle\pi(t) \pi\left(t_{0}\right)\right\rangle_{t},
$$

where $t_{0}$ and $t$ denote moments of time in the box, and correlator $\left\langle\pi(t) \pi\left(t_{0}\right)\right\rangle_{t}$ can be cast in the form

$$
\begin{aligned}
\left\langle\pi(t) \pi\left(t_{0}\right)\right\rangle_{t}= & \sum_{\substack{i, j=1 \\
i \neq j}}^{3} \frac{1}{3}\left[\lim _{t_{\max } \rightarrow \infty} \frac{1}{t_{\max }}\right. \\
& \left.\times \int_{t_{0}}^{t_{\max }} d t^{\prime} \pi^{i j}\left(t+t^{\prime}\right) \pi^{i j}\left(t^{\prime}\right)\right]
\end{aligned}
$$

with $\pi^{i j}$ being nondiagonal part of the stress-energy tensor $T^{i j}$

$$
\pi^{i j}(t)=\frac{1}{V} \sum_{i \neq j} \frac{p^{i}(t) p^{j}(t)}{E(t)} .
$$

Here $p^{i(j)}$ and $E$ are the $i(j)$ th components of momentum and energy of the particle, respectively. $t_{0}$ is the initial cutoff time indicating the beginning of the extraction of quantities from the box. The coefficient $1 / 3$ in the sum $\sum_{i, j}$ means averaging over the directions, which allows one to reduce the statistical errors. Usually the cutoff time $t_{0}$ is set to zero. We have left it here on purpose to explore the influence of the onset of data extraction from box calculations on the extracted value of shear viscosity.

If the system is in equilibrium, the correlator (10) is expected to experience an exponential drop with time, i.e.,

$$
\left\langle\pi(t) \pi\left(t_{0}\right)\right\rangle_{t}=\left\langle\pi\left(t_{0}\right) \pi\left(t_{0}\right)\right\rangle \exp \left(-\frac{t-t_{0}}{\tau}\right),
$$

with $\tau$ being an effective relaxation time of the system.

Inserting Eq. (12) in Eq. (9), one gets

$$
\eta\left(t_{0}\right)=\frac{\tau V}{T}\left\langle\pi\left(t_{0}\right) \pi\left(t_{0}\right)\right\rangle .
$$

As follows from Eq.(13), the problem of evaluation of $\eta$ is reduced to estimation of $\tau$. Shear viscosity may be obtained then in two different ways: (i) by direct calculation of integral from Eq. (9), which is equivalent to taking into account all time contributions to the correlator, or (ii) by fitting the correlator to Eq. (12) in some selected time interval and applying Eq. (13). The key difference here is the influence of fluctuations. The first case takes them into account and assumes that they are mostly mutually extinguished, whereas the second one cuts off fluctuations at times $t \gg \tau$ when the correlator is too small compared to the fluctuations (white noise) [11]. In what follows we compare the relaxation times $\tau$ for both cases.

\section{RESULTS}

First, we study the time evolution of the bulk characteristics in central cell of $\mathrm{Au}+\mathrm{Au}$ collisions at four energies in 

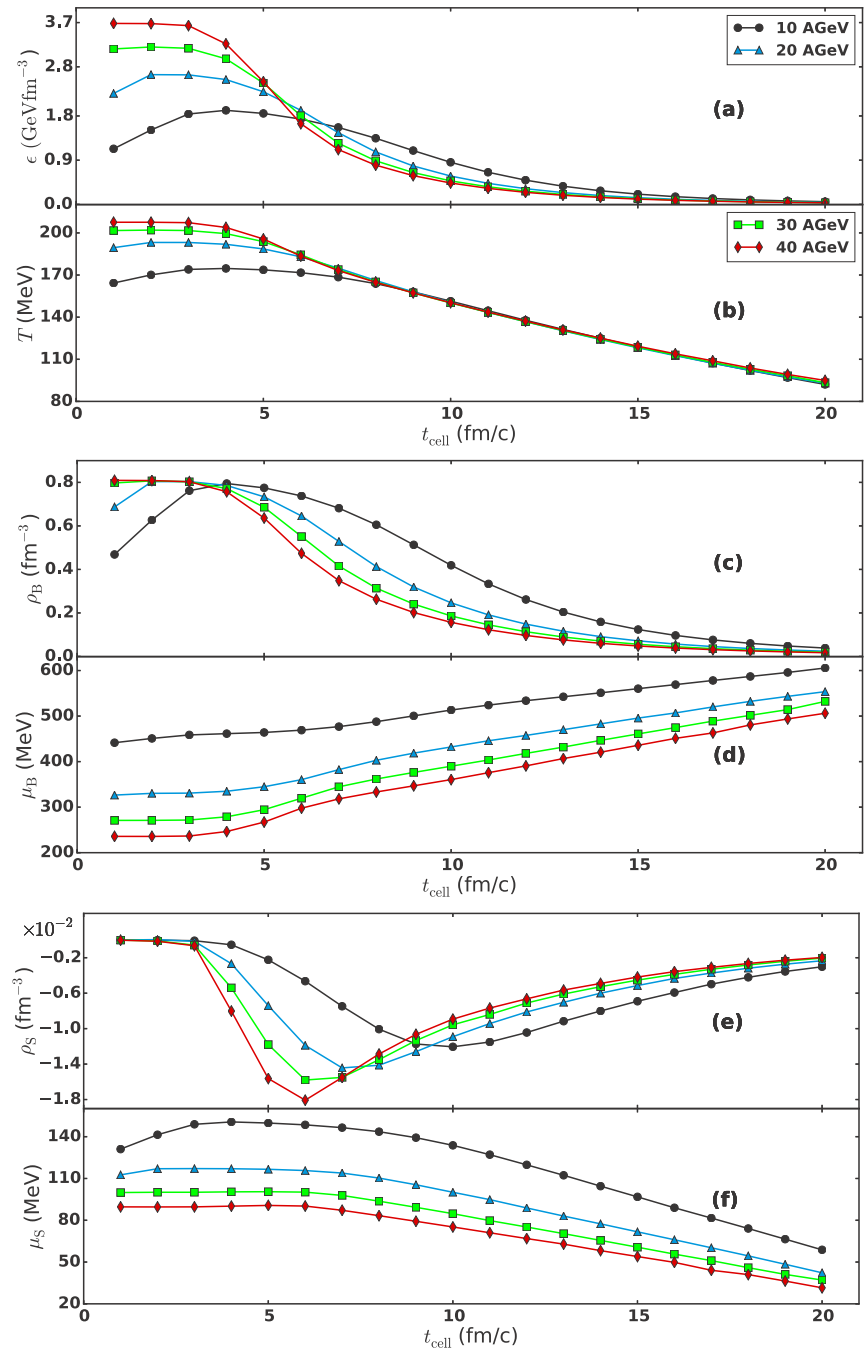

FIG. 1. Time evolution of (a) energy density $\varepsilon$, (c) net baryon density $\rho_{\mathrm{B}}^{\text {net }},(\mathrm{e})$ net strangeness density $\rho_{\mathrm{S}}^{\text {net }}$, (b) temperature $T_{\mathrm{SM}}$, (d) baryon chemical potential $\mu_{\mathrm{B}}$, and (f) strangeness chemical potential $\mu_{\mathrm{S}}$ in the central cell with $V=125 \mathrm{fm}^{3}$ in central $\mathrm{Au}+\mathrm{Au}$ collisions calculated within UrQMD at energies $E_{\mathrm{lab}}=10 \mathrm{~A} \mathrm{GeV}$ (circles), $20 A \mathrm{GeV}$ (triangles), $30 \mathrm{~A} \mathrm{GeV}$ (squares), and $40 A \mathrm{GeV}$ (diamonds). Lines are drawn to guide the eye.

question. Entropy density, net baryon density, and net strangeness density obtained in the cell from the microscopic calculations at time $1 \leqslant t \leqslant 20 \mathrm{fm} / c$ are displayed in Figs. 1(a), 1(c), and 1(e). At lowest bombarding energy $E_{\mathrm{lab}}=$ $10 A \mathrm{GeV}$ the maximum values of $\varepsilon$ and $\rho_{\mathrm{B}}$ are reached at $t \approx$ $5 \mathrm{fm} / c$, corresponding to complete overlap of two colliding nuclei. With rising bombarding energy, the nuclei overlap occurs earlier, thus the maxima of the distributions are shifted to times $t \approx 1-3 \mathrm{fm} / c$. With the net strangeness in the cell the situation is more peculiar. Copious production of strange particles takes place between $4 \mathrm{fm} / c$ and $8-10 \mathrm{fm} / c$ when the matter in the cell is baryon rich. As mentioned in [27-30], $K^{+}$'s can leave the selected volume a bit earlier compared to the $K^{-}$'s because of the smaller interaction cross sections. Therefore, the net strangeness in the cell is always negative, though small. Applying the procedure explained in Sec. III
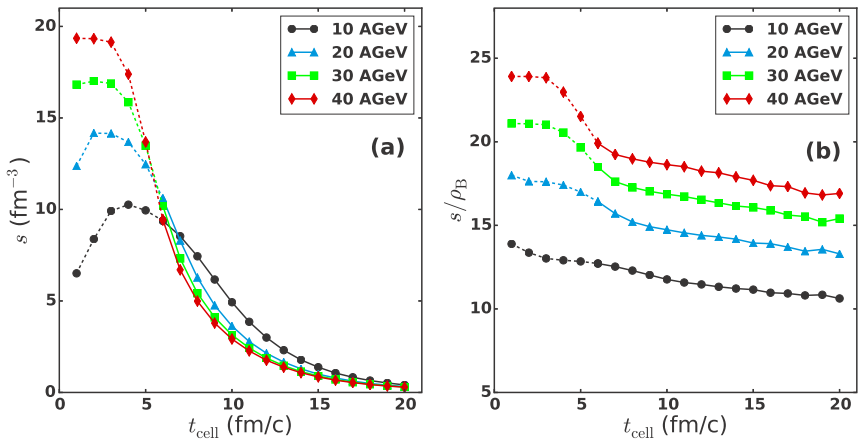

FIG. 2. (a) Entropy density $s_{\mathrm{sm}}$ and (b) its ratio to net baryon density $s_{\mathrm{sm}} / \rho_{\mathrm{b} \text {,net }}$ for different collision energies $E$ in UrQMD central cell calculations. Lines are drawn to guide the eye.

we insert the values of $\left\{\varepsilon, \rho_{\mathrm{B}}, \rho_{\mathrm{S}}\right\}$ as an input in the SM to get $\left\{T, \mu_{\mathrm{B}}, \mu_{\mathrm{S}}\right\}$ corresponding to an ideal hadron gas in chemical and thermal equilibrium. Evolutions of these parameters are shown in Figs. 1(b), 1(d), and 1(f). It is worth noting that the local equilibrium in the cell at energies between $10 A$ and $40 A \mathrm{GeV}$ is reached not earlier than $t \approx 6-8 \mathrm{fm} / c$. Therefore, one should treat the SM parameters obtained for earlier times with great care. Large baryon and energy densities observed at $t \leqslant 6 \mathrm{fm} / c$ are caused by interpenetration of two Lorentzcontracted nuclei. This leads to extra-high temperatures of the ideal hadron gas, seen in Fig. 1(b). For the extraction of more reliable values of $T$ and $\mu_{\mathrm{B}}$ we have to wait until the remnants of colliding nuclei will pass through each other and leave the tested volume. From here, we will indicate the thermodynamic results related to the early phase of the matter evolution in the cell by dashed lines in the figures.

Despite the differences in the cell initial conditions, all four temperature curves sit on the top of each other after $t=7 \mathrm{fm} / c$. Both baryon and strangeness chemical potentials drop with increasing bombarding energy, in full accord with the SM analysis of experimental data. However, $\mu_{\mathrm{B}}$ increases whereas $\mu_{\mathrm{S}}$ decreases, while the temperature in the cell drops and the matter becomes more dilute.

Figure 2(a) presents the evolution of the entropy density in the central cell in the studied reactions. This behavior is qualitatively similar to that of $\varepsilon(t)$ seen in Fig. 1(a). Note, however, that the entropy density here is calculated within the SM implying the maximum values for $s$. For the nonequilibrium state at $t \leqslant 6 \mathrm{fm} / c$ the entropy density is lower than the $s^{\mathrm{SM}}$. The ratio of entropy density to baryon density, $s / \rho_{\mathrm{B}}$, shown in Fig. 2(b), also should be lower during the stage of relaxation to equilibrium. It drops slightly, about $15 \%$ between 6 and $20 \mathrm{fm} / c$, indicating that the expansion proceeds nearly isentropically.

We are switching now to the box calculations. Figure 3 shows correlators defined by Eq. (10) calculated for all four collision energies. The input data $\varepsilon, \rho_{\mathrm{B}}, \rho_{\mathrm{S}}$ were extracted from the central cell of $\mathrm{Au}+\mathrm{Au}$ central collisions at times from $1 \mathrm{fm} / c$ up to $20 \mathrm{fm} / c$ after the beginning of the collision. To see the differences between the distributions more distinctly, each correlator was multiplied by the factor $10^{t_{c e l l}-1}$. Recall, that the results of the box calculations are shown for 

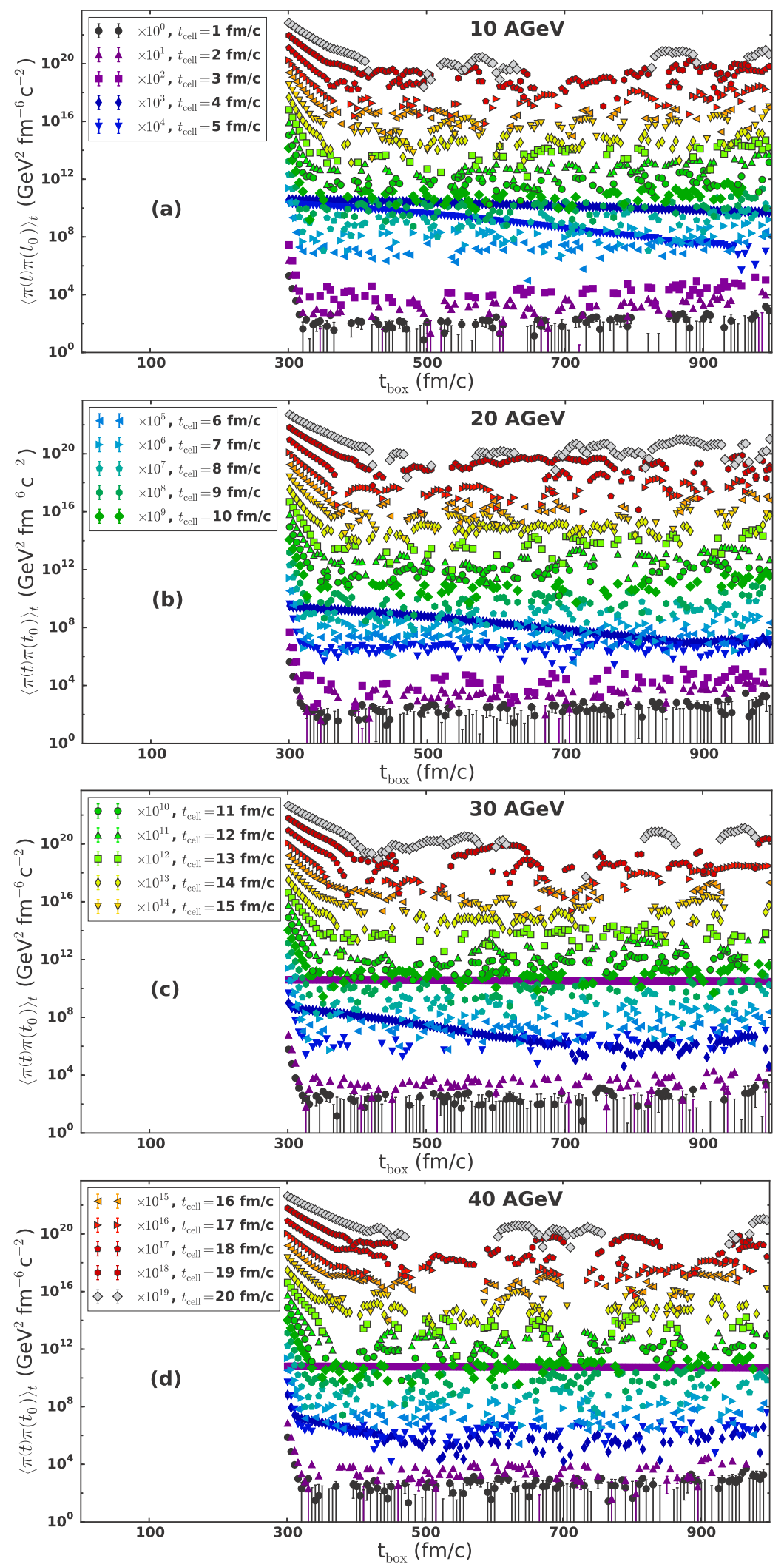

FIG. 3. Correlators $\left\langle\pi(t) \pi\left(t_{0}\right)\right\rangle_{t}$ for initial cutoff time $t_{0}=300 \mathrm{fm} / c$ in the UrQMD box calculations. Initial conditions for the boxes are taken from the central cell with $V=125 \mathrm{fm}^{3}$ of $\mathrm{Au}+\mathrm{Au}$ collisions at (a) $E_{\mathrm{lab}}=10 \mathrm{~A} \mathrm{GeV}$, (b) $20 \mathrm{~A} \mathrm{GeV}$, (c) $30 \mathrm{~A} \mathrm{GeV}$, and (d) $40 \mathrm{~A} \mathrm{GeV}$ at times $t=1-20 \mathrm{fm} / c$. Each distribution is multiplied by factor $10^{t_{\text {cell }}-1}$. 


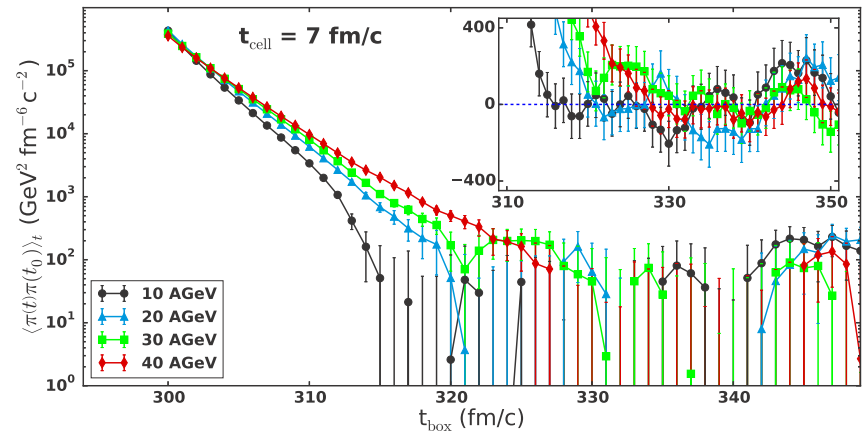

FIG. 4. UrQMD box calculations of the correlators $\left\langle\pi(t) \pi\left(t_{0}\right)\right\rangle_{t}$. Initial conditions in the box correspond to those in the central cell of $\mathrm{Au}+\mathrm{Au}$ collisions at $E_{\mathrm{lab}}=10 \mathrm{~A} \mathrm{GeV}$ (circles), $20 \mathrm{~A} \mathrm{GeV}$ (triangles), $30 \mathrm{~A} \mathrm{GeV}$ (squares), and $40 \mathrm{~A} \mathrm{GeV}$ (diamonds) taken at time $t_{\text {cell }}=7 \mathrm{fm} / c$. Lines are drawn to guide the eye.

times $t_{b o x} \geqslant 300 \mathrm{fm} / c$. This timescale has nothing to do with the typical relaxation times of hot and dense matter in heavyion collisions [24]. One can see that all correlators reveal exponential falloff with time in accordance with Eq. (12). However, for the conditions corresponding to early cell times, the relaxation rates are several orders of magnitude slower compared to those corresponding to late times. This cannot be explained entirely by large baryon and energy densities in the central cell at early $t_{\text {cell }}$, when nuclei overlap. Here one has to initialize the box with one or two very ultrarelativistic kaons that cannot redistribute their energy and momenta quickly enough. This circumstance results in a slow relaxation of the appropriate correlators. In order to extract the correct data corresponding to the overlap of nuclei, one has to process the box calculations for longer periods of time; see, e.g., [16]. Note also that microscopic transport models usually lack the inverse reactions to multiparticle processes $2 \rightarrow N(N \geqslant 3)$.
In this case the matter in the box will relax to the steady state rather than to the pure equilibrium; see, e.g., [23,24,31,32]. However, the matter in the central cell at $t \geqslant 6 \mathrm{fm} / c$ in heavyion collisions at energies below $E_{\text {lab }}=40 A \mathrm{GeV}$ becomes dilute very quickly. Its energy density drops, and the manyparticle inelastic reactions in the box with similar $\varepsilon, \rho_{\mathrm{B}}$, and $\rho_{\mathrm{S}}$ rapidly cease, thus leading to equilibrium similar to that of the SM.

At late times of the box calculations it appears that the correlations are rising. This is a technical effect. Namely, at the end of the UrQMD box calculations the program forces decay of all strongly decaying resonances, which may lead to some momentum correlations.

Typical behavior of the correlator dynamics on shorter timescales is demonstrated in Fig. 4, where the correlators for different collision energies are depicted. Again, as in Fig. 3, the initial cutoff time in the box is $t_{0}=300 \mathrm{fm} / c$. The initial conditions in the box correspond to that in the cell at $t_{\text {cell }}=7$ $\mathrm{fm} / c$. The exponential falloff with time occurs within $t \lesssim$ $t_{0}+30 \mathrm{fm} / c$. After that time the correlators become too weak, and fluctuations start to dominate the system. Domination of the fluctuations leads to the necessity of cutting off the dataset while fitting the correlator $\left\langle\pi(t) \pi\left(t_{0}\right)\right\rangle_{t}$ to Eq. (12), as was proposed in $[11,16]$.

The necessity for dataset cutoff raises the question of direct applicability of Eq. (9) in numerical calculations. In order to investigate the problem, we compare next the relaxation times extracted both from the integral in Eq. (9), $\tau_{\text {int }}\left(t_{0}\right)$, and by fitting the correlator to Eq. (12) within the time interval cutoff $t_{0} \leqslant t \leqslant\left(t_{0}+30\right) \mathrm{fm} / c, \tau_{\text {fit }}\left(t_{0}\right)$.

Figure 5 depicts the dependence of relaxation time $\tau_{\text {int }}$, extracted from the integral in Eq. (9), on the initial cutoff time $t_{0}$, with every tenth point being shown. As one can see, the relaxation usually takes a longer period for $t_{0}$ shorter than $200 \mathrm{fm} / c$ and vanishes for $t_{0} \geqslant 900 \mathrm{fm} / c$. For the initial times between these two limits the relaxation time

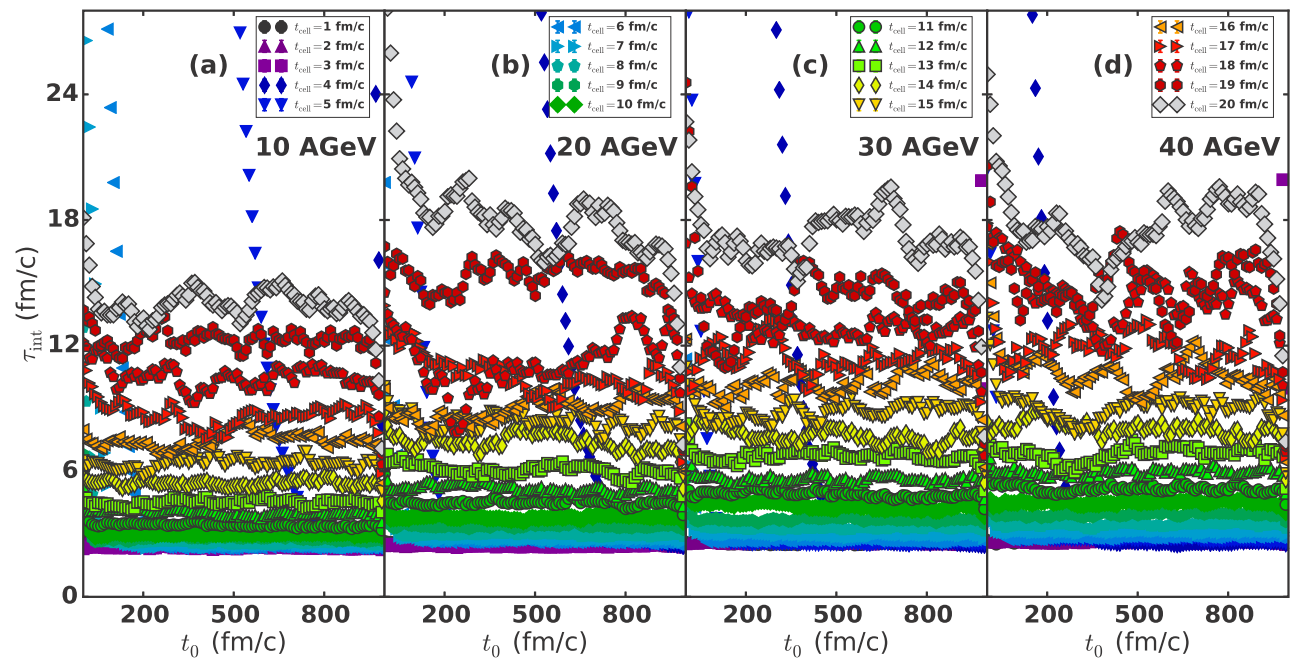

FIG. 5. Relaxation time $\tau_{\text {int }}\left(t_{0}\right)$ for the collision energies (a) $E_{\text {lab }}=10 A \mathrm{GeV}$, (b) $20 A \mathrm{GeV}$, (c) $30 A \mathrm{GeV}$, and (d) $40 A \mathrm{GeV}$ and for all cell times $1 \leqslant t_{\text {cell }} \leqslant 20 \mathrm{fm} / c$ in the UrQMD box calculations. 


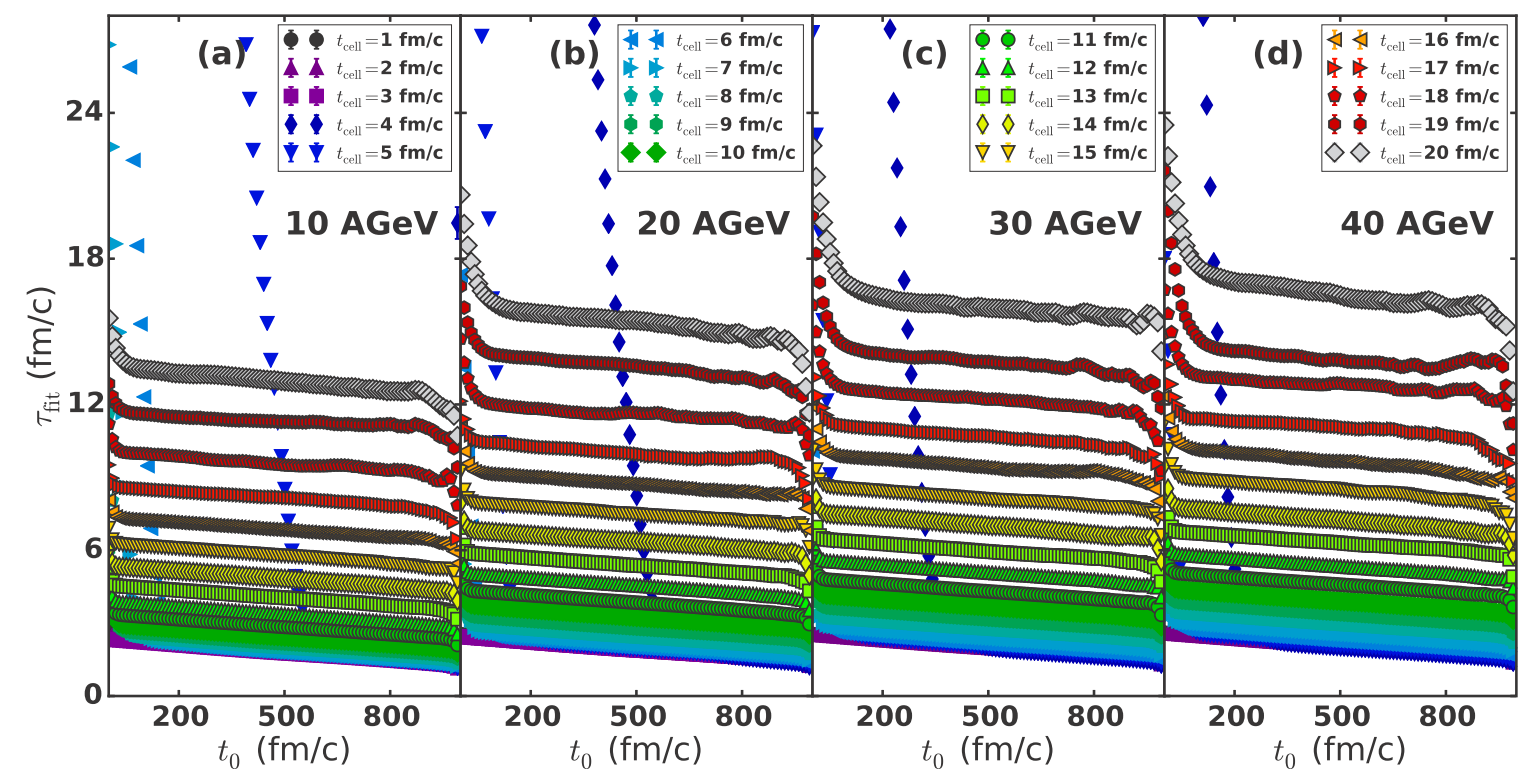

FIG. 6. The same as Fig. 5 but for relaxation time $\tau_{\mathrm{fit}}\left(t_{0}\right)$.

is rather constant. The only exceptions are at the early cell times.

Figure 6 displays the dependence of relaxation time $\tau_{\mathrm{fit}}$ extracted by fitting over the time interval $t_{\text {box }} \in\left[t_{0}, t_{0}+30\right]$. The behavior of $\tau_{\text {fit }}$ is pretty similar to that of $\tau_{\text {int }}$. However, the results presented in Fig. 6 have no stochastic oscillations, in contrast to those shown in Fig. 5. This can be explained by the influence of fluctuations on $\tau_{\text {int }}$. It is worth mentioning that, as one can notice, the plateau demonstrates some slope in Fig. 6 at $t_{0} \geqslant 200 \mathrm{fm} / c$ as compared to the results shown in Fig. 5. The slope may significantly influence the determination of $\eta$ values, because for early cell times with minimum values of $\tau_{\text {fit }}$ it may vary approximately by $40 \%$ for $200 \leqslant t_{0} \leqslant 800 \mathrm{fm} / c$. Small values of $\tau_{\text {int(fit) }}$ at large $t_{0}$ are dealing with the small averaging interval; see Eq. (10). Namely, the time resolution at large $t_{0}$ is too high to observe the correlator falloff, and one finds a kind of Brownian motion instead.

For the midrange of the initial cutoff time $t_{0}$ at the plateau-see Figs. 5 and 6 - the falloff rate does not change significantly. Thus, the values of $t_{0}$ from this range are well suited for our task. In the following we average the value of $\tau_{\text {int(fit) }}$ over the plateau in order to reduce statistical errors. Large values of the relaxation time $\tau_{\text {int/fit }}$ for some early cell times $t_{\text {cell }}$ are explained by the copious production of new hadrons and their subsequent rescatterings in very hot and dense baryon-rich matter at the very beginning of the collision. Additional time delay is caused by energetic single negative kaons. Combination of these factors forces the extension of the box calculations up to 2000 (sometimes 3000) $\mathrm{fm} / c$.

Figure 7 shows ratio of the relaxation times determined by Eqs. (9) and (12), $\left\langle\tau_{\text {int }}\right\rangle /\left\langle\tau_{\text {fit }}\right\rangle$. As we see, $\tau_{\text {int }}$ exceeds $\tau_{\text {fit }}$ by $25 \%$ at $t=6 \mathrm{fm} / c$. For the cell conditions at later stages the relaxation times converge and agree with each other within
$10 \%$ accuracy at $t \geqslant 15 \mathrm{fm} / c$. Thus, taking the fluctuations into account results in increase of $\tau$, as well as in its noiselike oscillations. The only difference, except for the general slope of $\tau_{\text {fit }}$, is observed at the early cell times, when the nuclei overlap.

Shear viscosity $\eta\left(t_{0}\right)$, calculated with $\tau_{\text {int }}$, is presented in Fig. 8. Since $\eta$ is proportional to $\tau_{\text {int }}$ due to exponential falloff behavior of the correlator, distributions in Figs. 5 and 8 have many similar features. Shear viscosity shows larger values for the initial box fluctuations at small times $t_{0}$. It is reduced significantly at large $t_{0}$, and has a plateau at intermediate times.

After averaging over the plateau, which we define as $t_{0} \in$ $[200,800] \mathrm{fm} / c$, one may obtain shear viscosity for different cell times at all the collision energies considered. Results are shown in Fig. 9. The statistical errors are smaller than the symbol sizes. We see that shear viscosity reaches its maximum

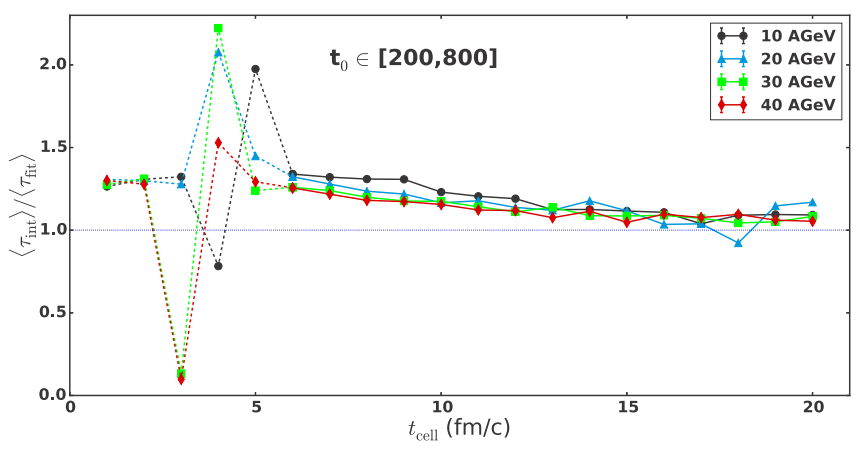

FIG. 7. Ratio $\left\langle\tau_{\text {int }}\right\rangle /\left\langle\tau_{\text {fit }}\right\rangle$ for the collision energies $10 A \mathrm{GeV}$ (circles), $20 A \mathrm{GeV}$ (triangles), $30 A \mathrm{GeV}$ (squares), and $40 A \mathrm{GeV}$ (diamonds) for all cell times $t_{\text {cell }}$. Errors are smaller than the symbol sizes. 


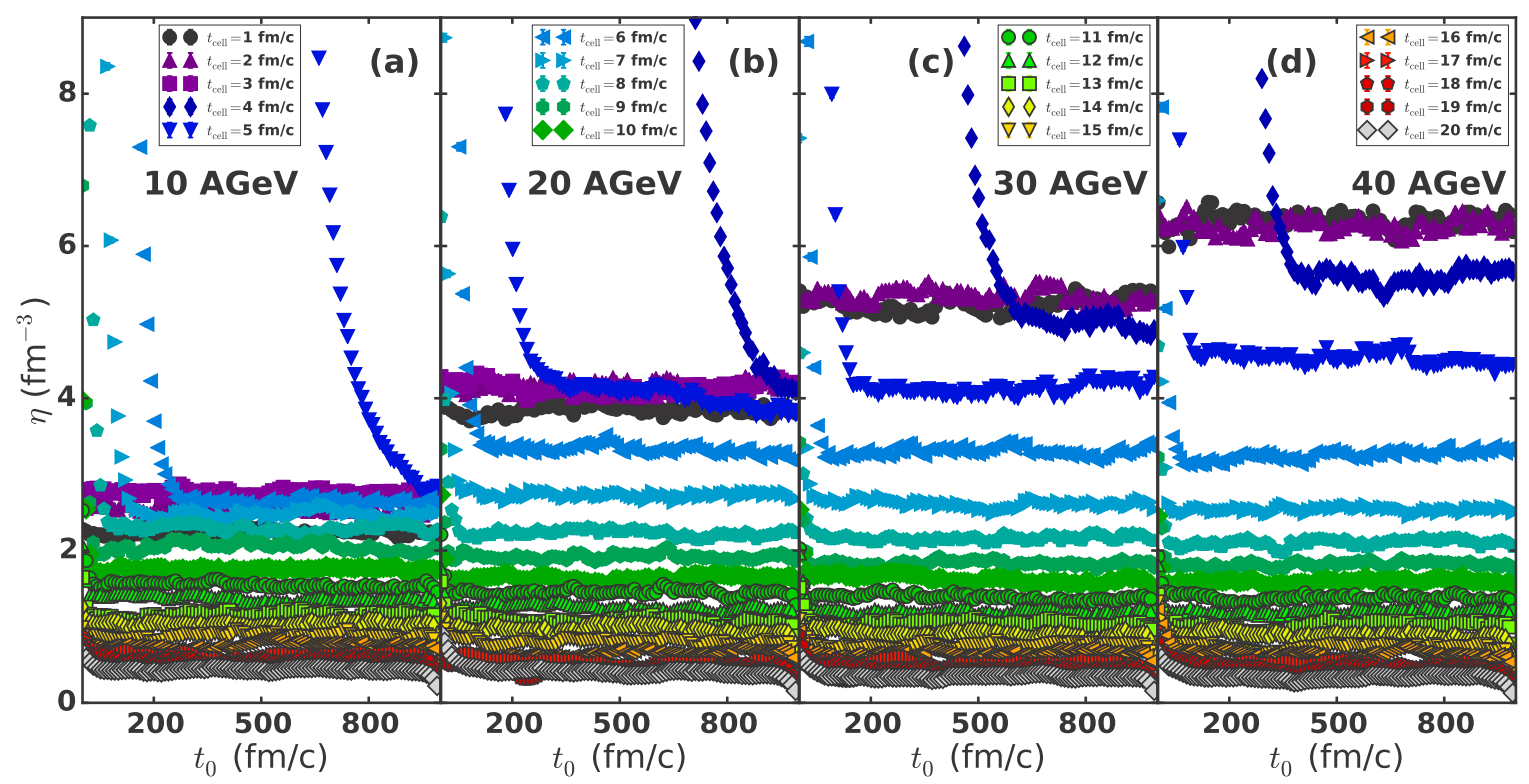

FIG. 8. Shear viscosity $\eta\left(t_{0}\right)$ for the collision energies (a) $E_{\mathrm{lab}}=10 \mathrm{~A} \mathrm{GeV}$, (b) $20 \mathrm{~A} \mathrm{GeV}$, (c) $30 \mathrm{~A} \mathrm{GeV}$, and (d) $40 \mathrm{~A} \mathrm{GeV}$ for all cell times $1 \leqslant t_{\text {cell }} \leqslant 20 \mathrm{fm} / c$ within the UrQMD box calculations.

at the very beginning of the heavy-ion collision. Then it gradually drops almost to zero at the late cell times. Decrease of $\eta$ with time is explained by the fact that at the late stages of the evolution of nuclear matter in the central cell there are only (quasi)elastic processes, i.e., soft scattering modes, remaining [29]. All energetic hadrons with large momenta have already left the cell. This circumstance results in the fast redistribution of momentum and energy of soft hadrons over the system, and, consequently, in small relaxation rate $\tau$ of the correlator.

At early times the shear viscosity is larger for heavy-ion collisions at larger energies. But after $t \approx 6 \mathrm{fm} / c$ all curves representing four different energies quickly converge. This behavior is very similar to the drop of the cell temperatures shown in Fig. 1(d). Both effects are caused by the faster loss of energy and baryon density in the central cell of central collisions with increasing bombarding energies.

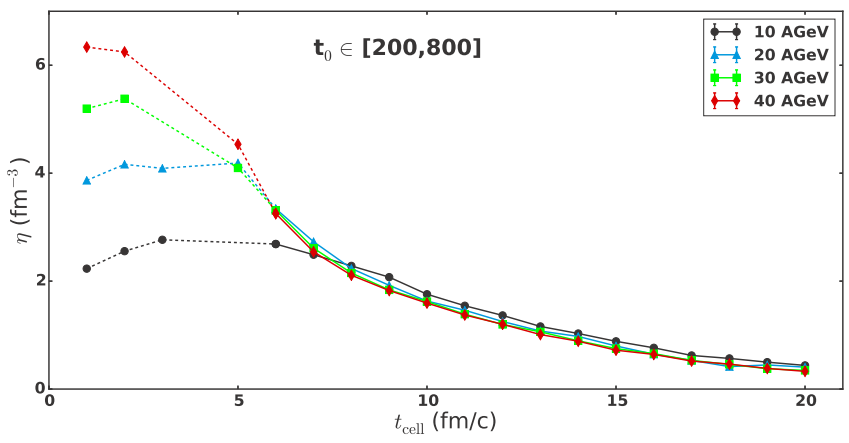

FIG. 9. Shear viscosity $\eta\left(t_{\text {cell }}\right)$ of hadrons in the central cell of central $\mathrm{Au}+\mathrm{Au}$ collisions at (a) $E_{\mathrm{lab}}=10 \mathrm{~A} \mathrm{GeV}$, (b) $20 \mathrm{~A} \mathrm{GeV}$, (c) $30 \mathrm{~A} \mathrm{GeV}$, and (d) $40 \mathrm{~A} \mathrm{GeV}$ within the UrQMD box calculations. Lines are drawn to guide the eye.
Finally, Fig. 10 displays $\eta / s$ dependencies on the evolution of the cell parameters, i.e., time [Fig. 10(a)], SM temperature [Fig. 10(b)], baryon chemical potential [Fig. 10(c)], and strangeness chemical potential [Fig. 10(d)]. The statistical errors are smaller than the symbol sizes. For all energies the ratio $\eta / s$ reaches its minimum at $t \approx 5 \mathrm{fm} / c$, when the nuclei are expected to overlap. Despite being small enough, the minima are about four times larger than the theoretical minimum value $1 / 4 \pi$. After that the ratio $\eta / s$ in the cell increases with time. The lower the collision energy, the smaller the ratio. It is also increasing with the drop of temperature and strangeness chemical potential, as shown in Figs. 10(b) and 10(d), and with the rise of baryochemical potential; see Fig. 10(c). It is worth noting that at $t \leqslant 5 \mathrm{fm} / c$ the matter in the cell is still out of equilibrium, whereas the estimates of $T, \mu_{\mathrm{B}}$, and $\mu_{\mathrm{S}}$ are done for fully a equilibrated system of hadrons. Therefore, all distributions at early times are indicated by the dashed curves.

Comparing our results to those calculated within the SMASH model in [16], one can notice a qualitatively different dependence of $\eta / s^{\mathrm{SM}}$ on the temperature. In contrast to the rise of $\eta / s$ with the temperature drop in the UrQMD cell calculations, SMASH demonstrates almost constant behavior of this ratio within the same temperature range. However, in the latter case the calculations were performed for a fixed baryon chemical potential, whereas in the UrQMD calculations it increases with the cell time $t_{\text {cell }}$. Another reason for deviations is the nonzero strangeness chemical potential in our calculations. Nevertheless, as shown in [16], the ratio $\eta / s$ increases in SMASH calculations with rise of baryon chemical potential, in accord with our results. Both UrQMD and SMASH indicate that shear viscosity decreases with decreasing temperature. This agreement is not accidental because of the conceptual similarity between UrQMD and SMASH. Further analysis concerning the influence of details of the system's internal dynamics, 

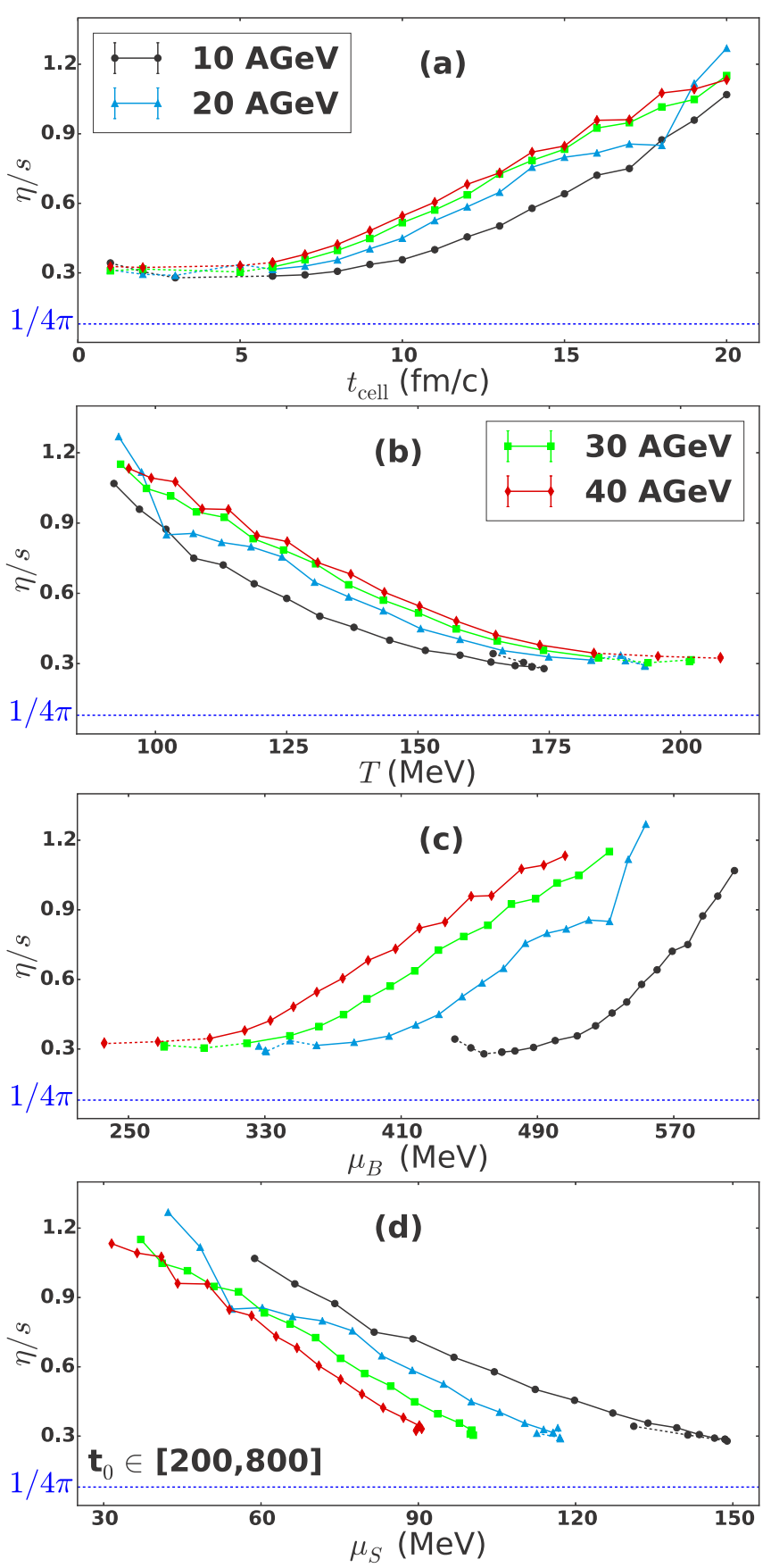

FIG. 10. Shear viscosity to SM entropy ratio $\eta / s_{\mathrm{sm}}$ as functions of (a) time, (b) temperature, (c) baryon chemical potential, and (d) strangeness chemical potential in the UrQMD calculations of central cell of central $\mathrm{Au}+\mathrm{Au}$ collisions at $E_{\mathrm{lab}}=10 \mathrm{~A} \mathrm{GeV}$ (circles), $20 A \mathrm{GeV}$ (triangles), $30 A \mathrm{GeV}$ (squares), and $40 A \mathrm{GeV}$ (diamonds). Lines are drawn to guide the eye. particularly, the role of lifetimes of resonances, on the $\eta / s$ ratio can be found in [16].

\section{CONCLUSIONS}

We have studied the shear viscosity of highly excited nuclear matter produced in the central area of central $\mathrm{Au}+\mathrm{Au}$ collisions at energies $E_{\mathrm{lab}}=10 \mathrm{~A}, 20 \mathrm{~A}, 30 \mathrm{~A}$, and $40 \mathrm{~A} \mathrm{GeV}$. Calculations are done within the UrQMD model. At the first stage, the energy density, the net baryon density, and the net strangeness density are determined for a cubic central cell with volume $V=125 \mathrm{fm}^{3}$. After that, the obtained values are used as input to the statistical model of an ideal hadron gas to calculate temperature, baryon chemical potential and strangeness chemical potential, as well as entropy density. The extracted values of $\varepsilon, \rho_{\mathrm{B}}$, and $\rho_{\mathrm{S}}$ are used also for initialization of the UrQMD box with periodic boundary conditions to study the relaxation of hot and dense nuclear matter to equilibrium. The Green-Kubo formalism is explored to calculate the shear viscosity.

It is shown that equilibrium in the box is achieved approximately after $t \geqslant 200 \mathrm{fm} / c$ for all but very high baryon and energy densities, corresponding to the overlap of the nuclei. The influence of initial cutoff time $t_{0}$ on momentum correlators is studied. Finally, the shear viscosity and its ratio to entropy density are calculated. We found that, for all four tested energies, $\eta$ and $s$ in the cell drop with time. Their ratios $\eta / s$, however, reach minima about 0.3 at $t \approx 5 \mathrm{fm} / c$, irrespective of the bombarding energy. Then the ratios rise to $\eta / s=1.0-1.2$ at $t=20 \mathrm{fm} / c$. This increase is accompanied by the simultaneous rise of baryon chemical potential and drop of both temperature and strangeness chemical potential in the cell.

\section{ACKNOWLEDGMENTS}

Fruitful discussions with K. Bugaev, Yu. Ivanov, D. Olynichenko, and O. Teryaev are gratefully acknowledged. The work of L.B. and E.Z. was supported by Russian Foundation for Basic Research (RFBR) under Grants No. 18-02-40084 and No. 18-02-40085, and by the Norwegian Research Council (NFR) under Grant No. 255253/F50, "CERN Heavy Ion Theory.” M.T., O.P., and O.V. acknowledge financial support of the Norwegian Centre for International Cooperation in Education (SIU) under grant "CPEA-LT-2016/10094 - From Strong Interacting Matter to Dark Matter." This work was also performed within the European network COST Action CA15213 "Theory of hot matter and relativistic heavy-ion collisions" (THOR). All computer calculations were made at Abel (UiO, Oslo) and Govorun (JINR, Dubna) computer cluster facilities.
[1] E. V. Shuryak, The QCD Vacuum, Hadrons and Superdense Matter, Lecture Notes in Physics, Vol. 71 (World Scientific, Singapore, 2004).

[2] L. P. Csernai, J. I. Kapusta, and L. D. McLerran, Phys. Rev. Lett. 97, 152303 (2006).
[3] P. K. Kovtun, D. T. Son, and A. O. Starinets, Phys. Rev. Lett. 94, 111601 (2005).

[4] P. Romatschke and U. Romatschke, Phys. Rev. Lett. 99, 172301 (2007)

[5] A. Muronga, Phys. Rev. C 69, 044901 (2004). 
[6] N. Demir and S. A. Bass, Phys. Rev. Lett. 102, 172302 (2009).

[7] P. Chakraborty and J. I. Kapusta, Phys. Rev. C 83, 014906 (2011).

[8] C. Wesp, A. El, F. Reining, Z. Xu, I. Bouras, and C. Greiner, Phys. Rev. C 84, 054911 (2011).

[9] S. Cremonini, Mod. Phys. Lett. B 25, 1867 (2011).

[10] J. Noronha-Hostler, J. Noronha, and C. Greiner, Phys. Rev. C 86, 024913 (2012).

[11] S. Plumari, A. Puglisi, F. Scardina, and V. Greco, Phys. Rev. C 86, 054902 (2012).

[12] V. Ozvenchuk, O. Linnyk, M. I. Gorenstein, E. L. Bratkovskaya, and W. Cassing, Phys. Rev. C 87, 064903 (2013).

[13] Iu. A. Karpenko, P. Huovinen, H. Petersen, and M. Bleicher, Phys. Rev. C 91, 064901 (2015).

[14] Yu.B. Ivanov and A. A. Soldatov, Eur. Phys. J. A 52, 117 (2016).

[15] J. Rais, K. Gallmeister, and C. Greiner, arXiv:1909.04522.

[16] J. B. Rose, J. M. Torres-Rincon, A. Schäfer, D. R. Oliinychenko, and H. Petersen, Phys. Rev. C 97, 055204 (2018).

[17] A. Motornenko, L. Bravina, M. I. Gorenstein, A. G. Magner, and E. Zabrodin, J. Phys. G 45, 035101 (2018).
[18] H. Song and U. Heinz, Phys. Rev. C 78, 024902 (2008).

[19] S. A. Bass et al., Prog. Part. Nucl. Phys. 41, 255 (1998).

[20] M. Bleicher et al., J. Phys. G 25, 1859 (1999).

[21] B. Andersson, G. Gustafson, and B. Nilsson-Almqvist, Nucl. Phys. B 281, 289 (1987).

[22] M. Tanabashi et al. (Particle Data Group), Phys. Rev. D 98, 030001 (2018).

[23] M. Belkacem et al., Phys. Rev. C 58, 1727 (1998).

[24] L. V. Bravina, E. E. Zabrodin, S. A. Bass, M. Bleicher, M. Brandstetter, S. Soff, H. Stöcker, and W. Greiner, Phys. Rev. C 62, 064906 (2000).

[25] M. S. Green, J. Chem. Phys. 22, 398 (1954).

[26] R. Kubo, J. Phys. Soc. Jpn. 12, 570 (1957).

[27] L. V. Bravina et al., Phys. Lett. B 434, 379 (1998).

[28] L. V. Bravina et al., J. Phys. G 25, 351 (1999).

[29] L. V. Bravina et al., Phys. Rev. C 60, 024904 (1999).

[30] L. V. Bravina et al., Phys. Rev. C 78, 014907 (2008).

[31] L. V. Bravina et al., Nucl. Phys. A 661, 600 (1999).

[32] E. L. Bratkovskaya, W. Cassing, C. Greiner, M. Effenberger, U. Mosel, and A. Sibirtsev, Nucl. Phys. A 675, 661 (2000). 